\title{
Informe sobre el 2011 Scientific Congress on Martial Arts and Combat Sports
}

Bruno Avelar-Rosa

Escola Superior de Educação. Instituto Politécnico de Viseu (Portugal)

Recibido 15/07/2011 - Aceptado 22/08/2011

\section{Resumen}

El presente informe resume las aportaciones realizadas en el 2011 Scientific Congress on Martial Arts and Combat Sports (2011 SCMACS), celebrado los días 13, 14 y 15 de mayo de 2011 en el Instituto Politécnico de Viseu (Portugal). En un ambiente caracterizado por una notable afluencia internacional, se expusieron un total de 36 comunicaciones y 25 pósteres, organizadas en ocho sesiones: 1) 0 bjeto de estudio, investigación y sistemática de las artes marciales y deportes de combate $(A M \& D C) ; 2$ ) Dimensiones pedagógica y didáctica en las AM\&DC; 3) Dimensión física de las $A M \& D C$; 4) Dimensiones física y técnica de las $A M \& D C ; 5)$ Dimensiones psicológica y táctica de las $A M \& D C ; 6)$ Dimensión socio-cultural de las $A M \& D C$; 7) Dimensiones socio-cultural y filosófica de las AM\&DC; y 8) Pósteres. En el ámbito de la recientemente creada Sociedad Científica Internacional de Artes Marciales y Deportes de Combate (IMACSSS) se organizó un área extraordinaria dedicada a la reunión de esta sociedad. Los resultados del congreso permiten observar el creciente interés de la academia en esta temática particular, así como el eminente desarrollo de la respectiva red intemacional.

Palabras clave: artes marciales, deportes de combate, investigación científica, reuniones científicas, congresos.

Report on the 2011 Scientific Congress on Martial Arts and Combat Sports

Abstract: The text resumes the communications and posters presented at the 2011 Scientific Congress on Martial Arts and Combat Sports (2011 SCMACS), held on 13, 14 and 15 May 2011, in the Polytechnic Institute of Viseu (Portugal). In an environment characterized by a significant international presence, a total of 36 communications and 25 posters were exposed, organized in eight sessions: 1) Study object, research and systematic of martial arts and combat sports (MA\&CS); 2) Pedagogical and didactics dimension on MA\&CS; 3) Physical dimensions on MA\&CS; 4) Physical and technical dimensions on MA\&CS; 5) Psychological and tactical dimensions on MA\&CS; 6) Sociocultural dimensions on MA\&CS; 7) Socio-cultural and philosophical dimensions on MA\&CS; and, 8) Posters. A formal meeting of the International Martial Arts and Combat Sports Scientific Society (IM ACSSS) also took place as an extraordinary event. The results 
of the congress gave us the possibility for observing the growing interest of the academy in this particular subject, as the evident development of an international network.

Key words: martial arts, combat sports, scientific research, scientific meetings, congresses.

Relatório sobre o 2011 Scientific Congress on Martial Arts and Combat Sports

Resumo: 0 presente texto resume as conclusões das comunicações e os posters apresentados(as) no 2011 Scientific Congress on Martial Arts and Combat Sports (2011 SCMACS), realizado nos dias 13, 14 e 15 de Maio de 2011, no Instituto Politécnico de Viseu (Portugal). Num ambiente caracterizado por uma notável afluência internacional, expuseram-se um total de 36 comunicações e 25 posters, organizados(as) em oito sessões: 1) Objecto de estudo, investigação e sistemática das artes marciais e desportos de combate $(A M \& D C)$; 2) Dimensões pedagógicas e didácticas em $A M \& D C$; 3) Dimensão física das $A M \& D C ; 3$ e 4) Dimensões física e técnica das $A M \& D C$; 5) Dimensões psicológica e táctica das $A M \& D C$; 6) Dimensão sócio-cultural das $A M \& D C$; 7) Dimensões sócio-cultural e filosófica das $A M \& D C ;$ e, 8) Posters. No âmbito da Sociedade Científica Internacional de Artes Marciais e Desportos de Combate (IMACSSS) recém criada, foi levada a cabo uma área extraordinária dedicada à reunião desta sociedade. Os resultados do congresso permitem observar 0 crescente interesse da academia nesta temática particular, assim como 0 eminente desenvolvimento da respectiva rede internacional.

Palavras-chave: artes marciais, desportos de combate, investigaçao científica, reuniões científicas, congressos.

Ponentes del Área no 6 - Dimensión socio-cultural de las $A M \& D C$. De izquierda a derecha: Raúl Sánchez

García (España), Raquel Escobar Molina (España), A bel Figueiredo (Portugal),

Zdenko Reguli (República Checa), Ana Rosa Jaqueira (Portugal)

y W ojciech Cynarski (Polonia).

Fotografía cortesía de Carlos Gutiérrez.

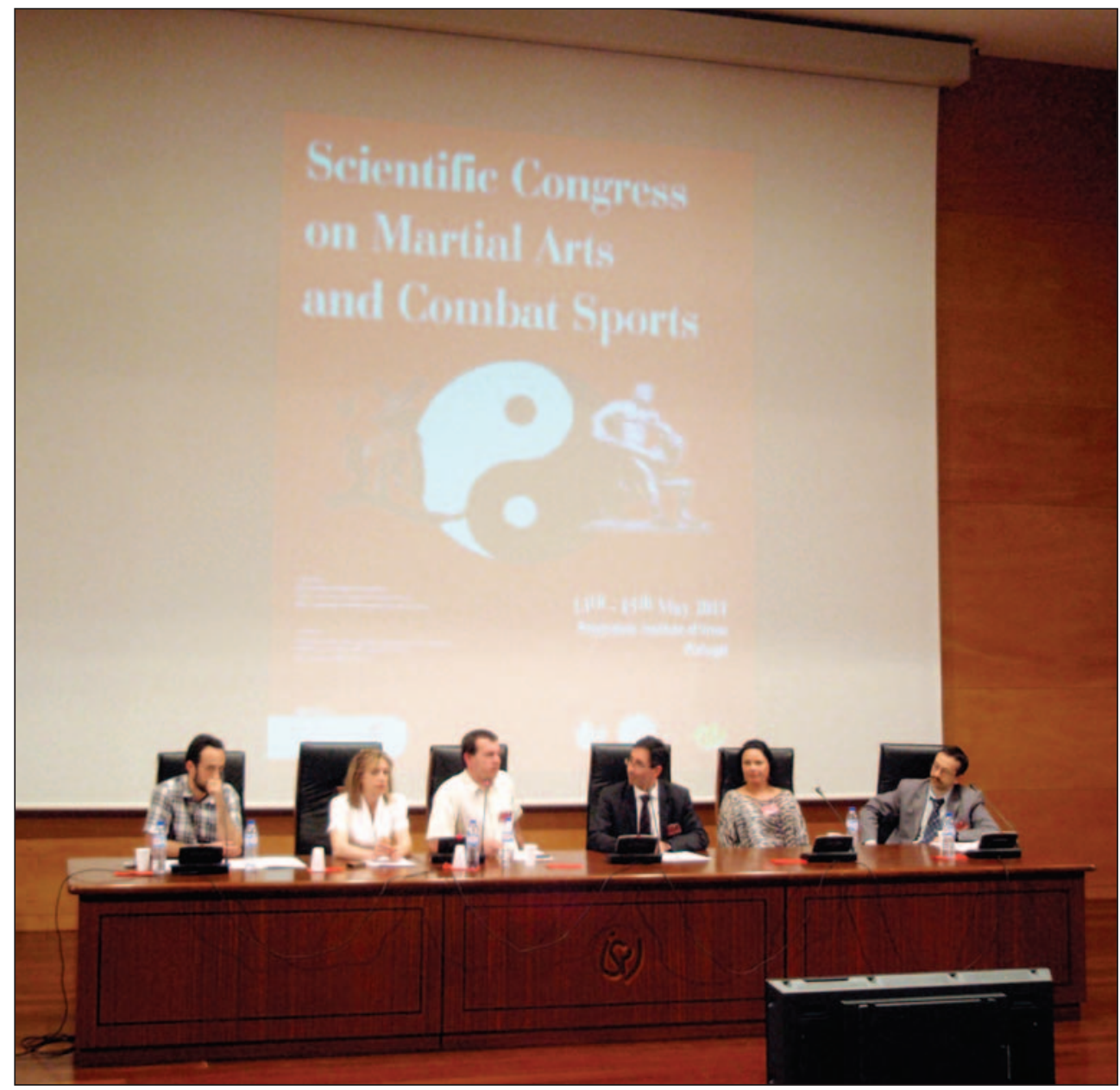

Avelar-Rosa $\triangle$ Informe sobre el 2011 Scientific Congress on Martial Arts and Combat Sports Revista de Artes Marciales A siáticas $\diamond$ Volumen 6 Número 2 (85-104) - 2011 


\section{Informe sobre el 2011 Scientific Congress on Martial Arts and Combat Sports}

Bruno Avelar-Rosa

Escola Superior de Educação. Instituto Politécnico de Viseu (Portugal)

\section{Introducción}

Por tercera vez, el Aula Magna del Instituto Politécnico de Viseu (IPV) acogió, entre los días 13 y 15 de mayo de 2011, la celebración del Scientific Congress on Martial Arts and Combat Sports (2011 SCMACS) ${ }^{1}$. El evento fue organizado por el Departamento de Ciências do Desporto e Motricidade de la Escola Superior de Educação de Viseu y la Associação para o Desenvolvimento e Investigação de Viseu (ADIV), contando además con la colaboración de otros organismos universitarios, deportivos y administrativos.

Asistieron al evento aproximadamente 50 participantes. 37 investigadores de 30 instituciones de enseñanza superior y 13 países (Brasil, China, España, Egipto, Estados Unidos, Filipinas, Francia, Irán, Italia, Nueva Zelanda, Polonia, Portugal, República Checa) presentaron un total de 69 comunicaciones, divididas en comunicaciones orales y pósteres. El idioma oficial del congreso fue el inglés. Tal como en anteriores ediciones, los objetivos generales del congreso fueron los siguientes:

a. Reunir a investigadores y especialistas para debatir el objeto de estudio de los contextos de las "artes marciales", "deportes de combate" o "defensa personal": la motricidad humana de combate;

b. Reflexionar sobre los resultados de las investigaciones (nacionales y extranjeras) en curso;

c. Divulgar proyectos de investigación relativos a las AM\&DC; y

d. Sensibilizar sobre la necesidad del estudio científico de las artes marciales y deportes de combate $(A M \& D C)$.

\footnotetext{
1 Puede consultarse el informe de la segunda edición del congreso en Rosa (2009).
} 
El comité científico del Congreso estuvo constituido por las siguientes personas.

\section{Comité Científico del 2011 SCMACS}

Dr. Abel Figueiredo (Instituto Politécnico de Viseu - Portugal)

Dra. Ágata Aranha (U. de Trás-os-M ontes e Alto Douro - Portugal)

Dra. Ana Rosa Jaqueira (U. de Coimbra - Portugal)

Dr. Andrew M. Lane (U. of W olverhampton - Reino Unido)

Dr. António Brito (Instituto Politécnico de Santarém - Portugal)

Dr. Benoit Gaudin (U. de Versailles Saint Quentin en Yvelines - Francia)

Dr. Carlos Gutiérrez-García (U. de León - España)

Dr. Cristiano Barreira (U. de São Paulo - Brasil)

Dr. Emerson Franchini. (U. de São Paulo - Brasil)

Dr. Eric Margnes (U. de Pau et des Pays de l'Adour - Francia)

Dr. Fumiaki Shishida (W aseda U. - Japan)

Dr. Helder Lopes (U. da Madeira - Portugal)

Dr. José Bragada (Instituto Politécnico de Bragança - Portugal)

Dr. Kai Filipiak (U. Leipzig - Alemania)

Dr. Keith Kernspecht (N. Sports Ac. \& Bulgarian State U. Plovdiv - Alemania)

Dr. King Yan Walter Ho (U. de Macau - China)

Dr. Marc Theeboom (Vrije Unversiteit Brussel - Bélgica)

Dr. Michel Calmet (U. Montepelier I - Francia)

Dra. Michela Turci (U. degli Studi di Milano - Italia)

Dr. Paulo Côelho Araújo (U. de Coimbra - Portugal)

Dr. Pierluigi Aschieri (U. degli Studi dell'Aquila - Italia)

Dra. Raquel Escobar Molina (U. de Granada - España)

Dr. Roman Kalina (U. Rzeszowski - Polonia)

Dr. Roberto Haramboure (U. Católica de Peru - Peru)

Dr. Samuel Julhe (U. Toulouse III - Francia)

Dr. Sergio Raimondo (U. degli Studi di Cassino - Italia)

Dr. Thomas Green (Texas A \& M U. - Estados Unidos)

Dr. W illy Pieter (Masarykova U., República Checa)

Dr. W ojciech Cynarski (U. Rzeszowski - Polonia)

Dr. Zdenko Reguli (Masarykova U. - República Checa)

La inauguración del congreso estuvo a cargo de Dña. Paula Carvalho, Vice-Presidenta del IPV, Dña. Cristina Gomes, Presidenta de la Escola Superior de Educação de Viseu, Dña. Paula Cardoso, responsable de uno de los centros de investigación del IPV, Dña. Teresa Oliveira, Directora del Departamento de Ciências do Desporto e Motricidade de la Escola Superior de Educação de Viseu y D. Guilherme Almeida, concejal de la Câmara Municipal de Viseu. Todos ellos destacaron, junto al responsable de la organización, Dr. Abel Figueiredo, la relevancia que ya tenía el congreso a pesar de sus únicamente tres años de historia, y se comprometieron nuevamente a seguir apoyando la iniciativa en futuras ediciones. 
Durante el congreso también se desarrolló la reunión anual de la International Martial Arts and Combat Sports Scientific Society (IMACSSS) [Sociedad Científica Internacional de Artes Marciales y Deportes de Combate].

A continuación se realizará un breve resumen de cada una de las comunicaciones presentadas en el congreso, organizadas según las diferentes áreas de discusión establecidas en el mismo. Aunque muchas de las comunicaciones resultan de un trabajo colectivo, en este informe únicamente haremos referencia al nombre del presentador. Para mayor información, remitimos a los lectores al libro de resúmenes del congreso (Figueiredo \& Gutiérrez-García, 2011).

\section{Área no 1 - Objeto de estudio, investigación y sistemática de las $A M \& D C$}

\section{Moderador: Abel Figueiredo (Instituto Politécnico de Viseu, Portugal).} Comunicaciones presentadas: 4.

ANÁLISIS BIBLIOMÉTRICO DELA PRODUCCIÓN CIENTÍFICA EN AM\&DC EN LAS BASES DE DATOS DE LA W EB OF SCIENCE (SCI-EXPANDED, $\mathrm{SSCl}, \mathrm{A} \& \mathrm{HCl})(2000-2009)$

Carlos Gutiérrez-García (Universidad de León, España)

En este trabajo se presentó el estado de la investigación científica en AM\&DC a través de la observación de los artículos publicados en las principales bases de datos de la W eb of Science en el periodo 2000-2009. Se hallaron un total de 1.527 artículos, los cuales pudieron ser organizados en 28 categorías. Los estudios sobre tai chi, judo, boxeo, lucha, qigong, kárate y genéricos fueron los más abundantes. Se pudieron detectar publicaciones en 583 revistas, en 13 idiomas y con referencia a 4.046 autores. Como aspectos más destacables, en los extremos del periodo considerado hubo un incremento del $354 \%$ en el número de artículos publicados, existen grandes diferencias entre determinados temas de investigación, e igualmente existe un claro predominio de las publicaciones realizadas en territorios de habla inglesa.

\section{AM VERNÁCULAS}

Thomas Green (Texas A \& M University, Estados Unidos)

En su presentación, Thomas Green comenzó enfatizando que las AM son sistemas de combate que implican aspectos más allá del componente físico, tales como la filosofía y la tradición. Seguidamente caracterizó las prácticas vernáculas, las cuales se refieren a aquellas propias de un lugar, tiempo 0 grupo, habiéndose desarrollado en contextos culturales y étnicos determinados, ilustrando con diversos ejemplos relativos a las AM. Las prácticas vernáculas tienen características comunes como son: legitimidad (interna y no impuesta), métodos de entrenamiento (integrados en el cotidiano del grupo), bricolaje (fluidez técnica), instalación (li- 
bre, sin escuelas), relación con la autoridad externa (apática u hostil) y currículo (no estructurado, lógica abierta, circunstancial). Son prácticas que habitualmente se muestran en contextos lúdicos y festivos, pero que mantienen una estructuración interna sólida, tangible y reconocible como AM efectivas.

CREANDO UN MARCO TIPOLÓGICO PARA LAS AM\&DC Joseph R. Svinth (Electronic Journals of Martial Arts and Sciences, Estados Unidos)

Joseph Svinth presentó un trabajo de sistematización de las AM, partiendo del hecho de que es necesaria una organización tipológica para aclarar y facilitar la comunicación en este campo de conocimiento. En este seguimiento, reveló la posible caracterización de las AM según sus atributos físicos (uso de implementos, características del espacio, indumentaria), regiones (local, global), rol social (profesionalizadas, participativas, vernáculas), uso (defensa, bienestar, etc.) o referencia temporal (atendiendo a sus diversas épocas de desarrollo hasta la actualidad).

TERMINOLOGÍA PREFERENTE PARA MEJORAR LA VISIBILIDAD DE LA LITERATURA CIENTÍFICA EN AM\&DC

Carlos Gutiérrez-García (Universidad de León, España)

En esta comunicación se señaló que la variedad terminológica utilizada en los trabajos científicos sobre AM \&DC dificulta su visibilidad y reconocimiento. Sobre la base del trabajo presentado anteriormente, se analizó esta problemática mediante indicadores bibliométricos, destacando aquellos términos más problemáticos. A partir de este análisis, el autor expuso algunas recomendaciones orientadas a mejorar la visibilidad de los artículos sobre $A M \& D C^{2}$.

\section{Área no 2 - Dimensiones pedagógica y didáctica en las AM\& DC}

Moderador: Sergio Raimondo (Università degli Studi di Cassino - Italia).

\section{Comunicaciones presentadas: 6.}

UN DEPORTE DE COMBATE - LA CAPOEIRA: SU APLICACIÓN EN EL CONTEXTO EDUCATIVO

Paulo Coêlho de Araújo (Universidade de Coimbra, Portugal)

Después de una breve contextualización histórica de la capoeira como modalidad deportiva, Paulo Côelho de Araújo identificó sus actuales formas de expresión, asumiendo que, alrededor de la lucha como eje central, se manifiestan el juego, el folklore, el deporte y la gimnasia. Este abanico conlleva que la capoeira posea un amplio potencial educativo debido a sus posibles aplicaciones artísti-

2 El texto completo de este trabajo ha sido publicado en Pérez-Gutiérrez; Gutiérrez-García y Escobar-M olina (2011) 
cas, rítmicas y melódicas, instrumentales, gimnásticas, lúdicas y gestuales. Entre estas posibilidades, fue destacado, en el ámbito de la educación física escolar, el potencial de las situaciones lúdicas y el eminente desarrollo de las habilidades gimnásticas como aspectos favorables para su práctica en la escuela.

\section{SABER LUCHAR GLOBAL - CARACTERIZACIÓN Y DESARROLLO}

Bruno Avelar-Rosa (Universitat de Girona, España)

Bruno Avelar-Rosa partió de la asunción de que existen contenidos técnicotácticos transversales en las disciplinas de combate, los cuales permiten definirlas como grupo. Tratando de caracterizar estos contenidos, el autor considera la existencia de unas acciones básicas (agarre, control, desequilibrio y toque), en estrecha relación con las situaciones ofensivas específicas de combate (golpeo, proyección, inmovilización, estrangulamiento y luxación). La adecuada aplicación de estas acciones en la dinámica del combate permite la manifestación de una intención táctica, la cual determina el logro del 'saber luchar'. Este concepto puede desarrollarse mediante modelos comprensivos de enseñanza-aprendizaje. El autor finalizó presentando la organización de su investigación de doctorado, la cual recoge este planteamiento teórico para su aplicación empírica en el ámbito escolar.

\section{RELACIONES TÉCNICO-TÁCTICAS EN LA EN SEÑANZA DE LOS DEPOR- TES DE COMBATE: IMPLICACIONES EN LA INICIACIÓN DEPORTIVA Mariana Gomes (Universidade de Campinas, Brasil \& Université de Tou- louse II, Francia)}

Mariana Gomes presentó su investigación sobre la importancia de la técnica y de la táctica en los procesos de iniciación a los deportes de combate, y las metodologías relacionadas con dicha iniciación. Para ello, realizó entrevistas semiestructuradas a cuatro profesores de $A M \& D C$, organizando el contenido declarado y analizado de acuerdo con tres categorías del conocimiento: conocimiento desde la práctica, a través de la práctica y para la práctica. En los resultados obtenidos la autora destacó, por un lado, la existencia de los tres tipos de conocimiento en los entrevistados, aunque estos no hayan demostrado estar articulados entre ellos, y, por otro lado, algunas contradicciones en el discurso de los profesores, los cuales, aunque hayan asumido la importancia de la utilización de metodologías comprensivas, evidenciaron el predominio de los aspectos vinculados a la ejecución técnica en sus métodos de enseñanza.

PROCESO DE EN SEÑ ANZA-APREN DIZAJE DE LAS HABILIDADES MOTORAS TÍPICAS DE LAS LUCHAS, AM\&DC

Emerson Franchini (Universidade de São Paulo, Brasil)

Emerson Franchini comenzó señalando que las técnicas de lucha fueron desarrolladas con propósitos específicos de acuerdo con el periodo histórico dominante. Sin embargo, en la actualidad, los ámbitos deportivo y educativo han impuesto nuevas perspectivas para el desarrollo de las técnicas marciales para 
garantizar su mejor aprendizaje y la seguridad de los participantes. Asimismo, se pasó de una enseñanza centrada en la imitación a una enseñanza que estimula la producción técnica a través de un abordaje centrado en la comprensión táctica. Estas metodologías, basadas en el modelo anglosajón del Teaching Games for Understanding, permiten el desarrollo de la creatividad y la búsqueda de nuevas soluciones ausente en los modelos de ejecución técnica. Sin embargo, el empleo de estas metodologías deberá ser compatible con las fases de aprendizaje de la técnica (cognitiva, asociativa y autónoma) y los principios pedagógicos del entrenamiento.

\section{EL ENFOQUE EDUCATIVO DE LA RELACIÓN MAESTRO-APRENDIZ EN}

\section{LAS AM VERNÁCULAS}

Zhang Guodong (Universidad del Sudoeste, China)

Zhang Guodong abordó la relación entre maestro y alumno en las AM vernáculas chinas. Esta es una relación que se basa frecuentemente en la genealogía familiar y se sustenta en rituales familiares que pasan de generación en generación. El autor presentó un caso concreto (Liangquang, o Boxeo de la Flor del Ciruelo) donde mostró los rituales vinculados a un maestro de 16 a generación respecto a los aprendices directos que tiene y de cómo esta certificación les incluye en la lógica familiar. En este ámbito, el aprendiz es una persona que ayuda a su maestro durante la vida cotidiana, y a su vez recibe soporte familiar de él, ya que el maestro posee una autoridad paternal sobre el alumno. El autor señala que esta forma de relacionarse vincula el proceso de enseñanza al aprendizaje para la vida, estimula la democracia entre iguales y fortalece el carácter. En esta línea, también las prácticas marciales asumen un carácter no deportivo, sino más bien higiénico, de desarrollo personal y de armonía social.

\section{EVALUACIÓN DEL APREN DIZAJE EN LA ENSEÑANZA DE DEFEN SA PER- SONAL EN INSTITUTOS DE EDUCACIÓN SECUNDARIA \\ Michal Vit (Masarykova Univerzita - República Checa)}

Michal Vit presentó el programa de defensa personal desarrollado en tres institutos de educación secundaria checos. Este programa consistió en la aplicación de un programa de entrenamiento durante 12 sesiones. Para la evaluación del programa fue utilizado un cuestionario para el dominio afectivo y simulación de situaciones de defensa personal para el dominio psicomotor. Según los resultados presentados, fue posible percibir que el alumnado reveló interés por el programa, que es más consciente del peligro, así como de la dificultad que conlleva el dominio de las situaciones de defensa personal. En el dominio psicomotor, el 50\% del alumnado no fue capaz de solucionar la situación de "agresión" simulada, evidenciando señales de estrés y ansiedad previas a la prueba. 


\section{Área no 3 - Dimensión física de las AM \& DC}

Moderador: Thomas Green (Texas A \& M University, Estados Unidos). Comunicaciones presentadas: 5 .

FUERZA ISOCINÉTICA DEL TOBILLO Y LA CADERA EN JÓVENES TAEKWONDISTAS

W illy Pieter (Masarykova Univerzita, República Checa)

En el estudio presentado por W illy Pieter participaron 23 hombres y 26 mujeres del equipo junior de taekwondo americano, para medir la fuerza isocinética concéntrica de las articulaciones del tobillo y de la cadera. Los resultados presentados revelaron las diversas relaciones entre las variables sexo, movimiento y velocidad angular. En sus conclusiones, el autor enfatizó la importancia de investigar las relaciones de la fuerza isocinética, fuerza en la ejecución de patadas y riesgo de lesión de los músculos isquiotibiales de los practicantes de taekwondo.

\section{ATLETAS OLÍMPICOS DE JUDO Y SUS RUTINAS DE ENTRENAMIENTO Emerson Franchini (Universidade de São Paulo, Brasil)}

En este estudio se aplicó un cuestionario a 57 judokas brasileños que participaron en los JJ.00. entre 1964 y 2008, referido a sus rutinas de entrenamiento (nage-komi, uchi-komi, randori, etc.) y de vida diaria (descanso, sueño, alimentación, ocio, etc.). Se analizaron las diferencias entre hombres y mujeres y entre medallistas y no medallistas. Como media, estos atletas dedicaron $23 \pm 8$ horas/ semana al entrenamiento, sin existir diferencias significativas en la gran mayoría de las categorías. Los resultados obtenidos mostraron diversas diferencias entre medallistas y no medallistas, así como entre hombres y mujeres, relativas a diversos componentes del entrenamiento, importancia asignada a los mismos 0 edades de iniciación y comienzo de la competición.

\section{FACTORES PREDISPONENTES DE LESIONES Y SU GRAVEDAD EN JUDO-}

KAS DE ELITE

Raquel Escobar Molina (Universidad de Granada, España)

El objetivo de este estudio fue determinar la influencia de la edad, el índice de masa corporal (IMC), el sexo, la experiencia en la alta competición y los métodos de control de peso en la aparición de lesiones y su respectiva gravedad en judokas de élite. La muestra fue de 144 judokas con edades comprendidas entre los 15 y 29 años, aplicándoseles diversos instrumentos de evaluación psicológica. Los resultados revelaron que los judokas con lesiones eran mayores, con más años en la alta competición y recurrían con mayor asiduidad a métodos de control de peso. Las mujeres que presentaron lesiones más graves presentaron un IMC más elevado y mayor frecuencia en el uso de métodos de control de peso. Este estudio permitió también concluir que los métodos utilizados por judokas para reducir el 
peso están basados particularmente en la abstinencia alimentaria y en la deshidratación, aumentando por ello el riesgo de sufrir lesiones y su gravedad.

\section{EL EQUILIBRIO Y LA RELACIÓN TIRAR-SEGAR-EMPUJAR EN JUDOKAS DE ELITE \\ Luís Monteiro (Universidade Lusófona de Humanidades e Tecnologias, Por- tugal)}

El objetivo de este estudio fue evaluar y comparar los perfiles de fuerza de hombres y mujeres judokas en relación a sus picos de fuerza explosiva, fuerza media y dinámica máxima de las extremidades superiores considerando la ratio agonista/antagonista. Fueron utilizados 42 atletas de elite de 5 países ( 30 hombres y 12 mujeres), los cuales fueron sometidos a los ejercicios de press de banca y remo. La ratio fue evaluada mediante una repetición máxima (1 RM) de ambos ejercicios. En general, los resultados obtenidos demostraron que las ratios de empujar y tirar son similares, y que existen diferencias significativas entre hombres y mujeres.

\section{ESTUDIO CLÍNICO PARA EVALUAR LOS BENEFICIOS TERAPÉUTICOS} DEL ENTRENAMIENTO DE TAIIIQUAN EN PACIENTES CON ENFERMEDAD DE PARKINSON IDIOPÁTICA

Sergio Raimondo (Università degli Studi di Cassino, Italia)

En este estudio se evaluaron los resultados inducidos por la práctica del taijiquan en una población de ocho personas mayores con enfermedad de Parkinson en relación a su marcha, equilibrio, postura y percepción subjetiva respecto a la mejora de su desempeño y bienestar. El grupo participó 5 veces a la semana en clases de 90 minutos de taijiquan durante 4 semanas. Los resultados obtenidos demostraron una mejora de la actividad motriz (particularmente del acto de caminar), así como de la percepción subjetiva de los pacientes y de sus escalas de vitalidad. Igualmente se manifestaron los problemas característicos de este tipo de estudios debido a la disponibilidad de la muestra.

\section{Área no 4 - Dimensiones física y técnica de las AM\& DC}

Moderador: W ojciech Cynarski (Uniwersytet Rzeszowski, Polonia). Comunicaciones presentadas: 6 .

\section{RETARDO ELECTROMECÁNICO EN ATLETAS DE KÁRATE}

António Brito (Instituto Politécnico de Santarém, Portugal)

Se analizó el retardo electromecánico en la ejecución de un movimiento balístico con los miembros superiores (golpe de puño de kárate gyaku-tsuki) comparando los valores alcanzados en hombres y mujeres. 10 hombres y 8 mujeres atletas de kárate realizaron 10 repeticiones de la técnica. El tiempo del movimiento, los instantes de inicio y final de la ejecución fueron registrados electromagnéti- 
camente. Los resultados obtenidos señalaron que solamente en el movimiento de flexión del brazo se verificó una diferencia significativa de los hombres respecto a las mujeres (menor retardo en los hombres).

\section{RESPUESTA ANTROPOMÉTRICA Y FISIOLÓGICA AL ENTRENAMIENTO} PERIODIZADO DE FUERZA EN ATLETAS DE KÁRATE

Emerson Franchini (Universidade de São Paulo, Brasil)

El objetivo de este estudio fue investigar las respuestas antropométricas y fisiológicas al entrenamiento de fuerza, e identificar en qué fases presentan los atletas su mejor desempeño. Participaron 14 atletas juveniles hombres en un programa de entrenamiento de fuerza durante 14 semanas ( 3 sesiones por semana). Se realizaron mediciones antropométricas y de la composición corporal, y pruebas de salto vertical y horizontal, press de banca y press de piernas. También se aplicó el test de W ingate y se midieron los valores de concentración de lactato en reposo e inmediatamente después de la práctica. Los resultados revelaron que no hubo alteración en las variables antropométricas y de composición corporal, press de banca y concentración de lactato. Sin embargo, el programa de entrenamiento produjo mejoría en los valores de la fuerza absoluta.

\section{PERFIL DE FUERZA ISOCINÉTICA DEL HOMBRO Y CODO EN ATLETAS}

DE JUDO

Rodrigo Ruivo (Universidade Técnica de Lisboa, Portugal)

En este estudio se caracterizó el perfil de fuerza de los miembros superiores en atletas de judo, a través de la evaluación isocinética de la rotación externa e interna del hombro y de la flexión y extensión del codo. 22 atletas de judo y un grupo control de 22 hombres sanos participaron en el estudio. Los resultados obtenidos señalaron mayores niveles de fuerza isocinética en los judokas, así como las similitudes y diferencias entre ambos grupos en las ratios de flexión/extensión. El autor finalizó realizando algunas recomendaciones prácticas para el entrenamiento en relación a la investigación realizada.

\section{ESTRÉS OXIDATIVO EN JUDOKAS FEMEN INAS DE ÉLITE} Raquel Escobar Molina (Universidad de Granada, España)

En este estudio se partió de la hipótesis de que las características físicas y fisiológicas del judo pueden afectar el sistema antioxidativo de los atletas. Se sometió a 10 judokas mujeres al test de Sterkowicz, registrando su frecuencia cardiaca y recogiendo dos muestras de sangre (al principio y 15 minutos después del test) a partir de las cuales se midieron indicadores de estrés oxidativo. Los resultados demuestran que el test aplicado causó estrés oxidativo en las judokas. A partir de estos resultados fue posible realizar individualmente recomendaciones específicas respecto al ajuste de las dietas y de ingestión de macronutrientes con vista al alivio del estrés oxidativo. 
En esta presentación, W illy Pieter analizó las lesiones ocurridas en jóvenes judokas filipinos. Se analizaron 192 judokas que compitieron en los campeonatos nacionales de judo en 2009. Los resultados obtenidos señalan que no hubo diferencia significativa en la ratio de lesiones entre judokas masculinos y femeninos. Comparando los resultados obtenidos con otros estudios, se aprecian diferencias significativas respecto a otros deportes de combate y países analizados.

FRECUEN CIA Y TIPO DE TÉCNICAS UTILIZADAS POR ATLETAS FEM ENINAS EN LA COMPETICIÓN DE TAEKW ONDO DE LOS JUEGOS PANAMERICANOS DE 2007

Emerson Franchini (Universidade de São Paulo, Brasil)

En este estudio se analizaron la frecuencia y el tipo de técnicas utilizadas por atletas de taekwondo en los juegos panamericanos de 2007, comparando los resultados de atletas medallistas y no medallistas. Se observaron 48 atletas en un total de 43 combates, categorizando las observaciones según las técnicas realizadas. Los resultados obtenidos revelan que todas las técnicas utilizadas para puntuar fueron patadas, y que las técnicas defensivas obtuvieron más puntuaciones que las técnicas ofensivas. No se encontró asociación entre el tipo de técnica y el éxito de los atletas.

\section{Área no 5 - Dimensiones psicológica y táctica de las AM\& DC}

Moderador: Walter Ho (Universidade de Macau, China). Comunicaciones presentadas: 4.

\section{ENSEÑ AR AUTOCONTROL A TRAVÉS DE LAS AM}

Eric Margnes (Université de Pau et des Pays de l'Adour, Francia)

Esta investigación tuvo por objetivo demostrar que la práctica de AM puede tener una influencia positiva en el auto-control e integración social. Las AM son prácticas que, desde su estructura filosófica, valoran el control emocional y de movimientos con vista a la adquisición de una eficiencia técnica y ética. Su tipología potencia la constante atención del profesor en la progresión del alumnado y el enfoque de estos en su propio comportamiento les ayuda al auto-control y a la responsabilidad en su proceso personal de aprendizaje. El autor expuso los resultados de dos investigaciones donde los practicantes de un arte marcial tradicional presentaron una mejora significativa de sus comportamientos pro-sociales, autoestima y nivel de agresividad en comparación con otros grupos de practicantes en contextos no tradicionales, donde no existe este modelo filosófico, el cual enfoca la transmisión de determinados valores sociales. 


\section{IDENTIDAD ATLÉTICA EN DEPORTES DE COMBATE}

Paulo Martins (Universidade Técnica de Lisboa, Portugal)

El objetivo de este trabajo fue investigar las relaciones entre la identidad atlética y las variables de género, edad y nivel competitivo en DC. Se utilizó el modelo explicativo de 5 dimensiones de Cieslak, el cual considera la exclusividad, la autoidentidad, la identidad social, la afectividad negativa y la afectividad positiva. Los resultados entre grupos de elite y recreación utilizados en el estudio señalaron una diferencia significativa en la dimensión de afectividad negativa (donde también se evidenciaron diferencias entre hombres y mujeres -mayor predominancia en estas-), demostrando que la identidad atlética parece estar vinculada a la identidad social y afectiva, y estar poco relacionada con un deporte en exclusiva.

\section{ACCIONES TÁCTICAS EN COMBATES DE ALTA COMPETICIÓN DE TAE- KWONDO: UN ESTUDIO DESCRIPTIVO \\ Cristina González de Prado (Universitat de Barcelona, España)}

Cristina González presentó un análisis observacional de 48 combates masculinos, realizados en las finales de 6 competiciones mundiales entre 2000 y 2007, orientado a caracterizar los movimientos propios de taekwondo de alto nivel. Entre otros resultados, se expuso la densidad de movimientos tácticos realizados por minuto y sus diferencias según categorías, la distribución de movimientos tácticos en los diversos asaltos, y las acciones de ataque, defensa y penalizaciones más frecuentes. A partir de estos resultados, se concluye que el análisis observacional sistemático puede ser una herramienta útil para que entrenadores y atletas optimicen los procesos de entrenamiento de cara a la competición.

\section{DIMENSIONES TÁCTICAS DE LA KATA: DESARROLLANDO LA INTELI-}

\section{GENCIA MOTRIZ EN AIKIDO}

Raúl Sánchez García (Universidad Europea de Madrid, España)

Raúl Sánchez abordó el interés en la utilización de la kata en el desarrollo de la inteligencia táctica en aikido. El autor se basa en la concepción de que la táctica implica la toma de decisiones ante la existencia de uno 0 más oponentes. Más aún cuando la realización de la kata en aikido implica necesariamente la presencia de un contrincante. La acción de este opositor (uke) deberá permitir los affordances (posibilidades, constreñimientos, orientaciones) necesarios para que se desencadene la acción plástica e inteligente por parte de aquel que realiza la kata (tori). Esto significa que determinadas acciones de uke deberán potenciar un abanico de posibilidades de respuesta por parte de tori. De esta manera, la kata como tarea más bien algorítmica deberá estar en relación continuada y bilateral con la práctica libre (jyu-waza) posibilitando el primero el entrenamiento de las diferentes posibilidades que se podrán dar en la situación de combate libre del segundo. La progresión y aumento de la complejidad y posibilidades tácticas deberán ser impartidas de acuerdo con el grado y nivel de los practicantes. 


\section{Área no 6 - Dimensión socio-cultural de las AM\& DC}

M oderadora: Raquel Escobar Molina (Universidad de Granada, España). Comunicaciones presentadas: 5.

VIAJAR PARA EL ESTUDIO DE LAS AM

W ojciech Cynarski (Uniwersytet Rzeszowski, Polonia)

W ojciech Cynarski señaló en su presentación que la existencia del fenómeno del turismo marcial merece ser estudiado. Se trata de practicantes de AM que, por razones de práctica, o simplemente para visitar locales emblemáticos de su $A M$, viajan a diferentes países, relacionándose con personas de otras procedencias y disfrutando de la oferta cultural de los países y ciudades donde se desplazan. Se trata de un flujo considerable de personas que va aumentando y que, hoy por hoy, se da también por razones científicas, tal como se ejemplifica en los últimos congresos científicos donde se presentan investigadores y practicantes de AM de todo el mundo.

\section{RITUALISMO EN EL MICROCOSMOS DE UN DOJO DE AIKIDO}

Zdenko Reguli (Masarykova Univerzita, República Checa)

Zdenko Reguli empezó su presentación considerando que el dojo de aikido es un microcosmos de la sociedad, donde existe también un gran formalismo y considerables comportamientos sociales realizados de forma ritualizada. Los objetivos de su estudio fueron comprender la organización típica de los entrenamientos de aikido, percibir otras realidades desde una perspectiva interna y observar diferencias entre rituales ideales (teóricos) y la realidad. Durante 3 meses, dos observadores relacionados (entre ellos el propio profesor) registraron diversos procesos sociales que sucedían en el dojo. De acuerdo con las observaciones realizadas, fue posible detectar símbolos propios del dojo de aikido, los cuales asumen diferentes significados para el practicante 0 el observador externo y que son seguidos por todos sin que sean impuestos.

\section{ANÁLISIS PRAXIOLÓGICO DEL PRIMER REGLAMENTO DEPORTIVO DE CAPOEIRA (1973) \\ Ana Rosa Jaqueira (Universidade de Coimbra, Portugal)}

Después de realizar una breve reseña histórico-institucional sobre el reconocimiento de la capoeira como deporte, la autora analizó el primer reglamento elaborado y asumido para esta modalidad desde un punto de vista praxiológico. El $81 \%$ de artículos de dicho reglamento hacían referencia a la lógica externa (aspectos históricos, legales, etc.) de la modalidad, mientras que las referencias a la lógica interna (relacionada con su caracterización técnica) sumaban aproximadamente el $19 \%$. Estos resultados revelan que en este reglamento la capoeira no fue todavía objeto de análisis desde el punto de vista de su praxis, por lo que no es posible 
establecer una estructura técnica sistematizada que caracterice internamente la modalidad y la defina como una disciplina deportiva particular.

AM\&DC Y LOS PROCESOS CIVILIZADORES

Raúl Sánchez García (Universidad Europea de Madrid, España)

Este trabajo estudió la aplicación de diferentes aspectos de la teoría de los procesos civilizadores de Norbert Elias a los DC/AM. Este análisis es posible a través de la relación entre los dominios sociogenéticos (procesos de formación de los Estados, poder figurativo) y psicogenéticos (hábitos personales). En esta línea, las prácticas de combate representan formas históricas de "violencia" actualmente institucionalizadas bajo las denominaciones de deporte 0 arte. Observando el hilo evolutivo de los DC/AM es posible detectar el paso de unas prácticas guerreras en una sociedad bélica a prácticas civiles con carácter simbólico desarrolladas en sociedades altamente pacificadas. Asimismo, las prácticas marciales actuales representan la evolución democrática (desde un punto de vista sociogenético) y los hábitos burgueses (desde un punto de vista psicogenético).

\section{DESAFIOS PARA LA FORMACIÓN DE ENTRENADORES DE AM\&DC} Abel Figueiredo (Instituto Politécnico de Viseu, Portugal)

En su presentación, A bel Figueiredo reflexionó sobre los contenidos que deberían ser considerados en la formación de entrenadores de AM \&DC. Basándose en el modelo de seis pasos de Patrick Duffy, enunció la importancia de reconocer el eje bio-psico-social como base multidimensional para la organización curricular. De esta forma, alrededor de las áreas científicas esenciales y de los factores de entrenamiento (referentes a los dominios físico, técnico, táctico, psicológico y socio-cultural) se plantean las aportaciones de la pedagogía, de la didáctica, de la teoría del entrenamiento y de la metodología del entrenamiento. Este modelo de formación permite entroncar con las exigencias internacionales de formación profesional y científica de entrenadores deportivos en general y de $A M \& D C$ en particular.

\section{Área no 7 - Dimensiones socio-cultural y filosófica de las AM \& DC}

M oderador: W illy Pieter (Masarykova Univerzita, República Checa). Comunicaciones presentadas: 6 .

EXPERIEN CIA VIVIDA EN CAPOEIRA - UN AM BRASILEÑO - A TRAVÉS DE LA FENOMEN OLOGÍA DEL CUERPO, TIEMPO, ESPACIO Y RELACIÓN HUMANA DE VAN MANEN

André Reis (Universidade de Brasília, Brasil)

En la teoría fenomenológica de Van Manen, el mundo es vivido en relación directa con lo que se experimenta diariamente. Para la definición del fenómeno 
vivido, se articulan cuatro conceptos: el cuerpo, el tiempo, el espacio y las relaciones humanas. En su investigación, André Reis presentó un análisis fenomenológico de la capoeira, realizado a partir de la observación participante, análisis de diarios de campo y entrevistas realizadas a 130 practicantes de capoeira. Los resultados obtenidos permitieron observar, analizar e interpretar formas particulares de vivenciar el cuerpo (sacrificio, habilidades, limitaciones, miedos), el tiempo (adherencia, persistencia, ganancias, mejoras), el espacio (interacción ritualizada, roles, el contexto familiar) y de las relaciones humanas (grupo social, conflicto, identidad, intimidad).

QUÉ ES EL COMBATE CORPORAL Y QUÉ ES EL ARTE MARCIAL: UNA RESPUESTA FEN OMEN OLÓGICA

Cristiano Barreira (Universidade de São Paulo, Brasil)

Basado en la fenomenología de Edmund Husserl, cuya intención es reducir cada objeto de observación a su esencia, Cristiano Barreira consideró en primera instancia que el combate corporal posee una evidencia estética, ya que se trata de una confrontación física. Esta confrontación puede asumirse como pelea (unilateral y motivada por la hostilidad), duelo (de común acuerdo entre ambos según una motivación respetuosa) o juego (enfocada al disfrute de la experiencia no hostil por ambos jugadores). La esencia del combate corporal señala la intención de sobreponerse al contrincante. Este objetivo está éticamente determinado por la actitud con que se vive la experiencia del combate. A la vez, las AM basan sus prácticas en el combate corporal, uniendo los polos éticos y estéticos considerados en este, a través de su entrenamiento.

\section{QUÉ HAY EN SUS MENTES: ANÁLISIS DE VARIOS GRUPOS DE ARTISTAS MARCIALES \\ Lenka Nejedlá (Masarykova Univerzita, República Checa)}

Este estudio nació del interés por entender las diferentes concepciones que los practicantes de AM desarrollan de acuerdo con las prácticas en que están implicados. Se preguntó a grupos de entre 23 y 30 practicantes de las disciplinas de aikido, aikibudo, muay thai y kárate qué significan estas prácticas para ellos. Posteriormente se desarrollaron mapas conceptuales basados en los términos clave utilizados por los entrevistados. Los resultados obtenidos revelaron una tendencia subjetiva e individual, presentando aspectos similares en las diferentes prácticas tales como la amistad, sacrificio o condición física, siendo el dolor el concepto mencionado más veces. Se destacó la ausencia de conceptos relacionados con el bienestar en el muay thai, o con el estilo de vida en el kárate.

\section{CÓMO ENTRENAR PARA UN COMBATE IMPUESTO}

Dominic Brizin (EW TO - European W ing Tsun Organization)

Siguiendo la idea del profesor Keith Kernspecht de que en las AM\&DC la forma deberá supeditarse a la función, Dominic Brizin señaló la importancia de 
analizar situaciones conflictivas reales para poder detectar los elementos a entrenar y aplicar en una lucha impuesta, ya que dichas situaciones presentan una estructura constante y común. En el entrenamiento se ha de considerar en primera instancia qué se puede hacer para evitar la agresión (prevención) y seguidamente qué se puede hacer para que el agresor no pueda combatir. Según esta teoría existen siete pasos fundamentales para la defensa personal: 1 . Conciencia/atención; 2. Flexibilidad; 3. Equilibrio; 4. Coordinación; 5. Escala de sentidos; 6 . 0 portunidad; y, 7. Espíritu guerrero.

NO SÓLO DANZA: ESTILO DE VIDA Y CULTURA EN EL FENÓMENO DE LAS BATALLAS URBANAS DE BAILE

Veronika Tumová (Masarykova Univerzita, República Checa)

Veronika Tumová analizó la evolución de las competiciones de batallas urbanas de baile (streetdance battles). La autora realizó una comparación entre los rituales y prácticas agonísticas existentes en estas danzas urbanas, cuya representación se da a través de la realización de coreografías con carácter técnico o a través de la comparación directa entre las habilidades de dos practicantes bajo la utilización del denominado freestyle (estilo libre). Aunque centrado en la calidad técnica del ejecutante, el carácter agonístico de los duelos freestyle acerca estas prácticas a los duelos realizados entre artistas marciales, toda vez que el desafío llevado a cabo está impregnado de una actitud guerrera por parte de los contrincantes.

\section{EL DESARROLLO DEL KÁRATE EN EL CONTEXTO DE LAS AM\&DC Abel Figueiredo (Instituto Politécnico de Viseu, Portugal)}

El objetivo de esta investigación fue analizar las motivaciones para la práctica del kárate, a través de un cuestionario aplicado a 244 practicantes avanzados portugueses de entre 13 y 71 años de edad. Se identificó la condición física, el bienestar psicológico y social, y la defensa personal como los factores valorados más importantes. La práctica de budo fue señalada como la orientación preferente, seguida de la práctica deportiva lúdica, ambas muy por encima de la orientación competitiva. Consecuentemente, el código budo fue destacado por el $66,8 \%$ de los practicantes como 'muy importante', de la misma manera que el $66 \%$ rechazó la realización de luchas como espectáculo (MMA, K1, PRIDE). El 63,5\% se manifestó totalmente en contra de la violencia. Los resultados obtenidos señalan diferentes percepciones, aunque exista una clara identificación y tendencias respecto a las prácticas asociadas al budo.

\section{Área no 8 - Pósteres}

Se presentaron un total de 25 pósteres. Por limitaciones de espacio, aquí presentamos únicamente el título de éstos y el primero de sus autores. 
- The non-selection of Fencing with the contents of physical education in schools in the Valencia Comunity. Ana Isabel Bastida.

- A study of Martial Arts in teaching for education and competition. Dong Xiaoxi.

- Taekw ondo to people with disabilities: concepts and perspectives. Proposed course: military personnel to the female gender violence. Mariana Gomes.

- Analysing of teaching methodologies used in the teaching of technique to beginners in striking Martial Arts and Combat Sports. Luís Preto.

- Relations between the Capoeira and the Martial Arts: implications in the scholar physical education. Gilbert dos Santos.

- Combat Sports in schools - an exploratory study in the Autonomous Region of Madeira. Miguel Vieira.

- Holistic development of young athletes in W restling. Sili Sue.

- The effects of detraining and training on an elite judoka after pregnancy. Abel Louro.

- A comparison of effort and pause in Judo matches. Bianka Miarka.

- Oxygen consumption and heart rate responses during Taekwondo combat simulation. Fábio Campos.

- Physiological and technical-tactical in Brazilian Jiu-jitsu competition. Gil Silva.

- Determination of food habits and weight changes in elite judoists by the restrained eating scale. Leonardo Andreto.

- Elite and sub-elite judokas: the factors behind international success. Luís Monteiro.

- Effects of energy intake restrictions and weight loss methods in a higher risk musculoskeletal injuries and changes in physiological status in elite. Rafael Ábalos.

- The roundhouse kick in function of the two target level. Coral Falcó.

- Effect of W ushu (Kung-Fu) training on manual dexterity. Margarida Santos

- The hemispheric preference relationship with the motor coordination in M MA athletes. Valécio Senna.

- Influence of environmental factors in top-level competition in Taekwondo combat. Cristina González del Prado.

- Combat sports and determinants of sport engagement in Portuguese athletes. Paulo Martins.

- Identification of the social characteristics of Capoeira fight practitioners in the cities of Coimbra and Aveiro. Evandro Lima.

- Social intervention of the Kickboxing coach. Gil Silva.

- Importance of submission sports (BJJ and Grappling) in Martial Arts. Jorge Lafuente.

- The value orientation of Martial Arts in modernization. Li Yun. 
- Challenge - A case study about a Portuguese kickboxing organization. Leonardo Cunha.

- Relational aspects betw een martial arts and Feldenkrais through movement. Juliana Souza.

\section{Área Extraordinaria - Reunión de la International Martial Arts and} Combat Sports Scientific Society - IMACSSS

En el 2011 SCMACS tuvo lugar una sesión abierta de la IMACSSS, sociedad creada en el año 2010. El presidente de la sociedad, W ojciech Cynarski, informó de la trayectoria y situación de la organización, observando los congresos científicos internacionales organizados desde su nacimiento (Rzeszów 2010 y Viseu 2011), y planteando una posible agenda científica hasta el año 2015. Destacó el surgimiento de divisiones científicas representativas, a través de las cuales se pretenden realizar investigaciones de forma articulada entre los diferentes miembros constituyentes de la sociedad. Igualmente, apuntó algunos proyectos ya en marcha.

La IMACSSS ya posee una página web (www.imacsss.com) y además está representada en varias revistas específicas.

Seguidamente se debatieron posibles líneas de desarrollo de la sociedad, señalando la necesidad de articular los ámbitos locales (o lingüístico-regionales) y globales.

La reunión terminó con la exposición de Sergio Raimondo sobre la organización del próximo congreso en Génova (Italia) en el año 2012. Tras esta exposición preliminar se recogieron las propuestas de los asistentes para mejorar dicho evento.

\section{Conclusiones}

La sesión de clausura del congreso fue realizada por Abel Figueiredo, quien destacó el crecimiento del nivel científico, la presencia de nuevos valores y la participación de investigadores no portugueses.

Desde nuestro punto de vista, este encuentro posibilitó el fortalecimiento de la comunidad científica internacional, así como el establecimiento de una red de contactos a escala global, de la cual podrán surgir futuras colaboraciones.

La dimensión internacional alcanzada por este congreso lanzó nuevos retos para el futuro, entre los cuales figura la articulación de la investigación local con las referencias globales, y la incentivación de nuevos investigadores y temáticas. Esta es una responsabilidad y motivación que fue asumida por aquellos que estuvieron presentes y que desarrollan su trabajo académico diario en instituciones de enseñanza superior.

Como se ha señalado, los resúmenes de las comunicaciones presentadas están disponibles en el libro de resúmenes del congreso. Se espera que algunas de las comunicaciones presentadas puedan aparecer publicadas en revistas científicas internacionales. 


\section{REFEREN CIAS}

Figueiredo, A.A. \& Gutiérrez-García, C. (Scientific Eds.) (2011). Proceedings of the Scientific Congress on Martial Arts and Combat Sports. Viseu - Portugal, 13th - 15th May 2011. Viseu: Associação para o Desenvolvimento e Investigação de Viseu, Escola Superior de Educação - Instituto Politécnico de Viseu.

Pérez-Gutiérrez, M.; Gutiérrez-García, C. y Escobar-Molina, R. (2011). Terminological recommendations for improving the visibility of scientific literature on martial arts and combat sports. Archives of Budo, 7(3), 159-166. Disponible en http://www.archbudo.com/fulltxt.php?ICID=881910. [Consulta 04/08/2011].

Rosa, V. (2009). Informe sobre el II Congresso Científico de Artes Marciais e Desportos de Combate. Revista de Artes Marciales A siáticas, 4(3), 102-115.

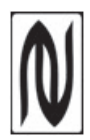

